\title{
Preliminary structural analysis of arch dams using a flat shell finite element formulation
}

\author{
S. Kömürcü*, M. Y1lmaz \\ Istanbul Technical University, Department of Civil Engineering, 34469, Istanbul, Turkey
}

\begin{abstract}
Arch dams have strong structural behavior because of their arch geometry. Numerical analysis of the arch dams is a necessary not only to validate the structural performance of the existing arch dams but also to model and analyze modern dam constructions. Finite element models for the arch dams are generally very complicated in terms of having many unknown parameters. In this study, finite element analysis of an arch dam is performed with using a shell finite element formulation to investigate the preliminary structural behavior of arch dams. A four-node shell finite element is proposed and used for analyzing of an arch dam. The arch dam geometry is simplified with using a middle surface. The formulation is verified with an arch dam example in the literature. Robustness of using a shell finite element formulation is indicated with the finite element analysis. Arch stresses at the upstream and downstream of the dam are presented as structural results. It is seen from the results, using a shell finite element is dramatically practical and effective for the structural analysis of the arch dams.
\end{abstract}

\section{Keywords}

Arch dams; Finite element method; Hydrostatic loading effect; Shell element; Static analysis

Received: 10 December 2018; Accepted: 19 March 2019

ISSN: 2630-5763 (online) C 2019 Golden Light Publishing® All rights reserved.

\section{Introduction}

The development of new finite elements has been a significant subject in recent years. Shell finite elements have a wide using area for engineering applications. The main purpose of producing new element formulations is to increase the success of the finite element analysis using as sparse as possible finite element mesh. Different approaches can be tried to produce a simple and effective shell finite element. One of the methods is to formulate a shell element with combining membrane element and plate bending element. This method is very preferred way of producing shell element because of the simplicity of the formulation.
Various studies have been carried out for the formulation of finite elements. Groenwold and Stander studied on the combinations of membrane and plate elements [1]. Bergan and Fellipa prepared a finite element formulation considering the out of plane displacement [2]. Cook combined a hybrid method with out of plane rotation [3]. Long and $\mathrm{Xu}$ studied to develop appropriate element models [4]. Pimpinelli worked on strain quadrilateral elements with drilling degree of freedom [5]. Y1lmaz et al. analyzed the geometrical nonlinear interaction of insulating glass panes with using plate element formulation [6]. Future work will be done to improve the results by making minor changes to existing element formulations.

\footnotetext{
Corresponding author

E-mail: komurcus@itu.edu.tr
} 
Arch dams have a significant place among the all dam types. Remarkable studies have been done to analyze of arch dams. Rydzewski produced a significant study on the theory of arch dams [7]. Bernardou and Boisserie used the thin shell theory to analyze arch dam structures [8].

In this study, the plane stress element with drilling degree of freedom and the plate bending element are combined to obtain a shell end element with 6 degrees of freedom in each node and 24 degrees of freedom totally. The finite element is used to perform a numerical experiment for the analyzing of arch dams effectively.

\section{Finite element formulation of shell element}

The shell finite element is obtained by combining the membrane element including the out of plane rotation and the plate bending element. Fig. 1 shows the combination of membrane and plate element to obtain the shell element.

$u_{c}, v_{c}$ and $w_{c}$ be the displacements in the local $x$, $y$ and $z$ directions, respectively. $\theta_{x}, \theta_{y}$ and $\theta_{z}$ are the rotations about the $x, y$ and $z$ axes, respectively. By writing the equilibrium equations in the $\mathrm{x}$ and $\mathrm{y}$ directions of the membrane element, the following equations are obtained by Eqs. (1) and (2). Where $N_{x}, N_{y}$ and $N_{x y}$ represent the $x, y$ and mixed component of the force, respectively and $q$ indicates the load.

$$
\begin{aligned}
& \frac{\partial N_{x}}{\partial x}+\frac{\partial N_{x y}}{\partial y}+q_{x}(x, y)=0 \\
& \frac{\partial N_{y}}{\partial y}+\frac{\partial N_{x y}}{\partial x}+q_{y}(x, y)=0
\end{aligned}
$$

Normal force and strain relationships can also be expressed in matrix form as shown in Eq. (3) where $E, v$ and $h$ are modulus of elasticity, Poisson ratio, and the thickness of plate, respectively.

$$
\left\{\begin{array}{l}
N_{x} \\
N_{y} \\
N_{x y}
\end{array}\right\}=\frac{E h}{\left(1-v^{2}\right)}\left[\begin{array}{ccc}
1 & v & 0 \\
v & 1 & 0 \\
0 & 0 & \frac{1-v}{2}
\end{array}\right]\left\{\begin{array}{l}
\frac{\partial u_{c}}{\partial x} \\
\frac{\partial v_{c}}{\partial y} \\
\frac{\partial u_{c}}{\partial y}+\frac{\partial v_{c}}{\partial x}
\end{array}\right\}
$$

The field terms are chosen for $u$ and $v$ are expressed as follows using $\alpha$ and $\beta$ coefficients in local coordinates $(s, t)$.

$$
\begin{aligned}
u(s, t)= & \alpha_{1}+s \alpha_{2}+t \alpha_{3}+s t \alpha_{4}+t^{2} \alpha_{5} \\
& +s t^{2} \alpha_{6}+t^{3} \alpha_{7}+s t^{3} \alpha_{8} \\
v(s, t)= & \beta_{1}+s \beta_{2}+t \beta_{3}+s t \beta_{4}+s^{2} \beta_{5} \\
& +t s^{2} \beta_{6}+s^{3} \beta_{7}+t s^{3} \beta_{8}
\end{aligned}
$$

Considering $(-1,-1),(1,-1),(1,1)$ and $(-1,1)$ in local coordinate values, respectively for the nodes $1,2,3,4$ of the rectangular element, after some mathematical manipulations the shape functions $\mathbf{N}_{\mathbf{u}}$ and $\mathbf{N}_{\mathbf{v}}$ can be found as follows;

$$
\mathbf{N}_{\mathbf{u}}=\frac{1}{8}\left[\begin{array}{c}
s\left(-t^{3}+3 t-2\right)+t^{3}-3 t+2 \\
b(s-1)(t+1)(1-t)^{2} \\
s\left(t^{3}-3 t+2\right)+t^{3}-3 t+2 \\
b(-s-1)(t+1)(1-t)^{2} \\
s\left(-t^{3}+3 t+2\right)-t^{3}+3 t+2 \\
b(-s-1)(t-1)(1+t)^{2} \\
s\left(t^{3}-3 t-2\right)-t^{3}+3 t+2 \\
b(s-1)(t-1)(1+t)^{2}
\end{array}\right]
$$

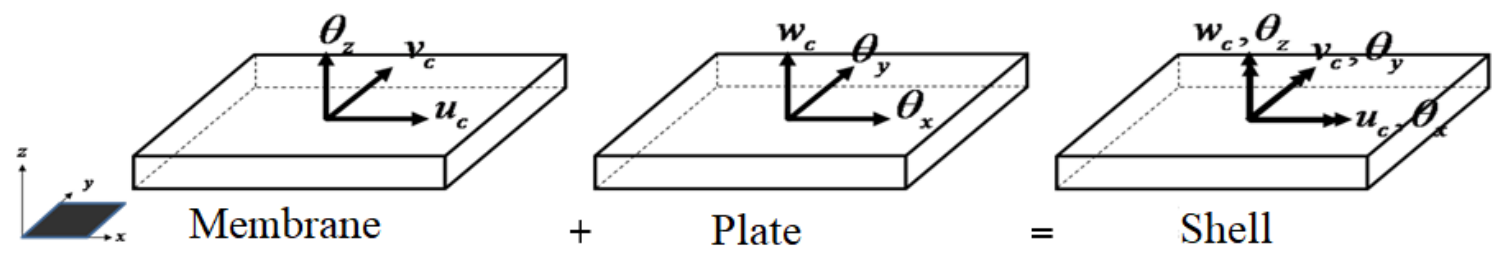

Fig 1. Shell element obtained by the combination of membrane and plate elements 


$$
\mathbf{N}_{\mathbf{v}}=\frac{1}{8}\left[\begin{array}{c}
s^{3}(1-t)+s(3 t-3)-2 t+2 \\
a(s+1)(1-s)^{2}(1-t) \\
s(3-3 t)+s^{3}(t-1)-2 t+2 \\
a(s-1)(1+s)^{2}(1-t) \\
s(3+3 t)+s^{3}(-t-1)+2 t+2 \\
a(s-1)(1+s)^{2}(1+t) \\
s(-3 t-3)+s^{3}(t+1)+2 t+2 \\
a(s+1)(1+t)(1-s)^{2}
\end{array}\right]
$$

The following is the classical plate theory available in [9]. By writing the equilibrium equations in the $x$ and $y$ directions for the plate, the following equations are obtained as Eqs. (8), (9) and (10). Where $V$ and $M$ are shear force and moment, respectively.

$$
\begin{gathered}
\frac{\partial V_{x}}{\partial x}+\frac{\partial V_{y}}{\partial y}+q(x, y)=0 \\
V_{y}=\frac{\partial M_{x y}}{\partial x}+\frac{\partial M_{y}}{\partial y}=0 \\
V_{x}=\frac{\partial M_{x y}}{\partial y}+\frac{\partial M_{x}}{\partial x}=0
\end{gathered}
$$

If Eqns. (9) and (10) are substituted in Eq. (8),

$$
\frac{\partial^{2} M_{x}}{\partial x^{2}}+2 \frac{\partial^{2} M_{x y}}{\partial x \partial y}+\frac{\partial^{2} M_{y}}{\partial y^{2}}+q(x, y)=0
$$
the governing differential equation is obtained as

Moment curvature relations can also be expressed in matrix form as Eq. (12). Where $D$ is the flexural rigidity of plate.

$$
\left(\begin{array}{l}
M_{x} \\
M_{y} \\
M_{x y}
\end{array}\right)=-D\left(\begin{array}{ccc}
1 & v & 0 \\
v & 1 & 0 \\
0 & 0 & \frac{1-v}{2}
\end{array}\right)\left(\begin{array}{l}
\frac{\partial^{2} w}{\partial x^{2}} \\
\frac{\partial^{2} w}{\partial y^{2}} \\
2 \frac{\partial^{2} w}{\partial x \partial y}
\end{array}\right)
$$

The differential equation is a fourth-degree equation at the Kirchhoff plate theory. To obtain a confined element the continuity of the displacements and slope must be realized. But it is difficult to obtain a confined and simple Kirchhoff plate element because of the mathematical foundation of the requirements are dramatically strict. Thus, unconfined plate elements are generally used instead of the confined elements. In this study, to obtain a confined plate element BFS (Bogner, Fox and Schmit) plate element is used based on Kirchhoff plate theory [10]. BFS plate element has second-degree mixed displacement derivations besides $\mathrm{x}$ and $\mathrm{y}$ displacements and rotations about $\mathrm{x}$ and $\mathrm{y}$ axes. Therefore, the plate element has 4 degree of freedom and totally 16 degree of freedom.

To derive the shape functions at the 16 degree of freedom plate element, field equations, having 16 coefficients, are chosen as follows in local coordinates $(s, t)$;

$$
\begin{aligned}
w(s, t) & =a_{1}+s a_{2}+t a_{3}+s^{2} a_{4}+s t a_{5}+t^{2} a_{6}+s^{3} a_{7}+s^{2} t a_{8}+s t^{2} a_{9}+t^{3} a_{10}+s^{3} t a_{11}+s^{2} t^{2} a_{12}+s t^{3} a_{13} \\
& +s^{2} t^{3} a_{14}+s^{3} t^{2} a_{15}+s^{3} t^{3} a_{16}
\end{aligned}
$$

$$
\theta_{x}=\frac{\partial w}{\partial y}=\frac{1}{b}\left(a_{3}+s a_{5}+2 t a_{6}+s^{2} a_{8}+2 s t a_{9}+3 t^{2} a_{10}+s^{3} a_{11}+2 s^{2} t a_{12}+3 s t^{2} a_{13}+3 s^{2} t^{2} a_{14}\right.
$$

$$
\left.+2 s^{3} t a_{15}+3 s^{3} t^{2} a_{16}\right)
$$

$$
\begin{aligned}
\theta_{y}= & -\frac{\partial w}{\partial x}=-\frac{1}{a}\left(a_{2}+2 s a_{4}+t a_{5}+3 s^{2} a_{7}+2 s t a_{8}+t^{2} a_{9}+3 s^{2} t a_{11}+2 s t^{2} a_{12}+t^{3} a_{13}\right. \\
& \left.+2 s t^{3} a_{14}+3 s^{2} t^{2} a_{15}+3 s^{2} t^{3} a_{16}\right) \\
w_{x y}= & \frac{1}{a b}\left(a_{5}+2 s a_{8}+2 t a_{9}+3 s^{2} a_{11}+4 s t a_{12}+3 t^{2} a_{13}+6 s t^{2} a_{14}+6 s^{2} t a_{15}+9 s^{2} t^{2} a_{16}\right)
\end{aligned}
$$


Considering $(-1,-1),(1,-1),(1,1)$ and $(-1,1)$ in local coordinate values, respectively for the $1,2,3,4$ nodes of the rectangular element, after some mathematical manipulations the plate shape functions $\mathbf{N}_{\mathbf{p}}$ can be obtained as follows;

$$
\mathbf{N}_{\mathbf{p}}=\frac{1}{16}\left[\begin{array}{c}
s^{3}\left(t^{3}-3 t+2\right)+s\left(-3 t^{3}+9 t-6\right)+2 t^{3}-6 t+4 \\
b\left(s^{3}-3 s+2\right)(1+t)(1-t)^{2} \\
a\left(s^{3}-s^{2}-s+1\right)\left(-t^{3}+3 t-2\right) \\
a b(s+1)(t+1)(1-s)^{2}(1-t)^{2} \\
s^{3}\left(-t^{3}+3 t-2\right)+s\left(3 t^{3}-9 t+6\right)+2 t^{3}-6 t+4 \\
b\left(-s^{3}+3 s+2\right)(1+t)(1-t)^{2} \\
a\left(s^{3}+s^{2}-s-1\right)\left(-t^{3}+3 t-2\right) \\
a b(s-1)(s+1)^{2}(1+t)(1-t)^{2} \\
\left.s^{3}-3 t-2\right)+s\left(-3 t^{3}+9 t+6\right)-2 t^{3}+6 t+4 \\
b\left(-s^{3}+3 s+2\right)(t-1)(t+1)^{2} \\
a\left(s^{3}+s^{2}-s-1\right)\left(t^{3}-3 t-2\right) \\
a b(s-1)(t-1)(1+s)^{2}(1+t)^{2} \\
s^{3}\left(-t^{3}+3 t+2\right)+s\left(3 t^{3}-9 t-6\right)-2 t^{3}+6 t+4 \\
b\left(s^{3}-3 s+2\right)(t-1)(1+t)^{2} \\
a\left(s^{3}-s^{2}-s+1\right)\left(t^{3}-3 t-2\right) \\
a b(s+1)(1-s)^{2}(t-1)(1+t)^{2}
\end{array}\right]
$$

The finite element equations can be obtained by using Virtual Work Theorem as Eq. (18).

$$
\begin{aligned}
& \iint_{A}\left(N_{x} \frac{\partial \bar{u}}{\partial x}+N_{x y}\left(\frac{\partial \bar{u}}{\partial y}+\frac{\partial \bar{v}}{\partial x}\right)+N_{y} \frac{\partial \bar{v}}{\partial y}\right) \mathrm{d} A \\
& +\iint_{A}\left(\frac{\partial \bar{w}}{\partial x}\left(\frac{\partial w}{\partial x} N_{x}+\frac{\partial w}{\partial y} N_{x y}\right)+\frac{\partial \bar{w}}{\partial y}\left(\frac{\partial w}{\partial x} N_{x y}+\frac{\partial w}{\partial y} N_{y}\right)\right) \mathrm{d} A \\
& -\iint_{A}\left(M_{x} \frac{\partial^{2} \bar{w}}{\partial x^{2}}+2 M_{x y} \frac{\partial^{2} \bar{w}}{\partial x \partial y}+M_{y} \frac{\partial^{2} \bar{w}}{\partial y^{2}}\right) \mathrm{d} A=\iint_{A}(\bar{w} q(x, y)) \mathrm{d} A
\end{aligned}
$$

The $12 \times 12$ membrane element system stiffness matrix $\mathbf{K}_{\mathbf{M}}$ is obtained by combining the $8 \times 8$ membrane element stiffness matrix with adding the $4 \times 4$ out of plane rotation matrix Eq. (19).

$$
\mathbf{K}_{\mathrm{M}}=\left[\begin{array}{cc}
\mathbf{K}_{8}^{u_{c}, v_{c}} & \mathbf{K}_{8,4} \\
\mathbf{K}_{4,8} & \mathbf{K}_{4}^{\theta_{z}}
\end{array}\right]
$$

The $12 \times 12$ stiffness matrix for the plate element $\mathbf{K}_{\mathbf{P}}$ is obtained by neglecting the $4 \times 4$ mixed $w_{x y}$ derivatives matrix as shown Eq. (20).

$$
\mathbf{K}_{\mathrm{P}}=\left[\begin{array}{cc}
\mathbf{K}_{12}^{w_{c}, \theta_{x}, \theta_{y}} & \mathbf{K}_{12,4} \\
\mathbf{K}_{4,12} & \mathbf{K}_{4}^{w_{c y}}
\end{array}\right]
$$

The $24 \times 24$ system stiffness matrix $\mathbf{K}$ of the shell element is obtained by combining the stiffness matrices of the membrane element with the plate element as shown in Eq. (21).

$$
\mathbf{K}=\mathbf{K}_{\mathrm{M}}+\mathbf{K}_{\mathrm{P}}=\left[\begin{array}{ccc}
\mathbf{K}_{12}^{u_{c}, v_{c}, \theta_{z}} & \mathbf{0} & \mathbf{0} \\
\mathbf{0} & \mathbf{K}_{12}^{w_{c}, \theta_{x}, \theta_{y}} & \mathbf{K}_{12,4} \\
\mathbf{0} & \mathbf{K}_{4,12} & \mathbf{K}_{4}^{w_{c y}}
\end{array}\right]
$$

\section{Numerical arch dam example}

The finite element formulation is applied to the Grand Maison Arch Dam. They used the linear thin shell theory and conforming finite elements. The arch dam geometry is simplified with using a middle surface. Thus, the dam can be modelled using plane finite element obtained in this study. Using plane elements instead of volume elements is a practical way of modelling huge structures such as dams. Analyze time considerably decrease using plane finite elements because of the degree of freedom of the system is decreased.

\subsection{Model properties}

The material parameters used for the static analysis of the arch dam; Modulus of elasticity is $\mathrm{E}=2 \times 10^{4}$ $\mathrm{MPa}$ and Poisson ratio is $v=0.2$. These parameters are the elastic material parameters of the dam. The geometrical properties are used as expressed in the study of [8]. The boundary conditions for the model are shown in Fig. 2. Fixed ends represent the contact surfaces between the arch dam and soil surface. Free end represents top of the arch dam. Only $1 / 2$ part of the dam is modelled in terms of symmetry using symmetrical boundary conditions. Using symmetrical boundary conditions is decrease the computational cost of the analyze effectively. 


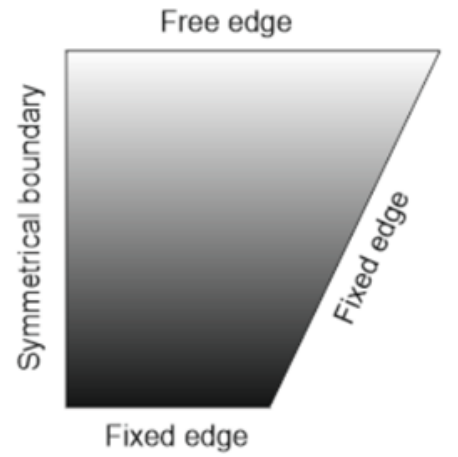

Fig 2. Boundary conditions of the dam

\subsection{Result of the finite element analysis}

To test the performance of the element, Grand Maison Arch Dam is modelled, and stress values are analyzed. The stress values measured at the top of the crown cantilever and base of crown cantilever. Upstream and downstream stress values are calculated as shown in Table 1. Only hydrostatic loading is considered for the finite element analysis. The stress values are compared with the study of Bernardou and Boisserie in 1982 [8]. The stress values resulted from gravitational forces is obtained as seen in Table 2 .

It can be stated that, the results are compatible with their study in terms of the theory and finite element models. In fact, the detailed analysis of an arch dams is done with considering the changing of thickness of the dam. It can be performed with taking changing cross-sections of the arch dam. Hence, using a middle surface for the analysis will be give us preliminary information for the arch dams.

\section{Conclusion}

In this study, a shell finite element having six degrees of freedom at each node and total of twenty-four degree of freedom is obtained. The membrane and plate element are combined to produce the shell element. Finite element analyzes are performed on the arch dam to investigate the performance of the shell element. As seen the compatible results with the Grand Maison arch dam example, the obtained shell element can be used for other similar arch dams making some simplifications such as using a middle surface. Although these results are not the exact results for the arch dam structures, the formulation can be used to perform a preliminary analysis on the arch dams to decide approximate values of their design parameters to make a detailed structural analysis.

Table 1. Stress values caused by hydrostatic load for the arch dam (MPa)

\begin{tabular}{lcccc}
\hline \multirow{2}{*}{ Study } & \multicolumn{2}{c}{ Top of cantilever } & \multicolumn{2}{c}{ Base of cantilever } \\
\cline { 2 - 5 } & Upstream & Downstream & Upstream & Downstream \\
\hline Bernardou and Boisserie [8] & -4.800 & -2.883 & 2.753 & -2.974 \\
Present study & -4.955 & -3.175 & 2.965 & -3.156 \\
\hline
\end{tabular}

Table 2. Stress values caused by gravitational load for the arch dam (MPa)

\begin{tabular}{lcccc}
\hline \multirow{2}{*}{ Study } & \multicolumn{2}{c}{ Top of cantilever } & \multicolumn{2}{c}{ Base of cantilever } \\
\cline { 2 - 5 } & Upstream & Downstream & Upstream & Downstream \\
\hline Bernardou and Boisserie [8] & -1.374 & -1.512 & -1.107 & -0.142 \\
Present study & -1.395 & -1.575 & -1.135 & -0.185 \\
\hline
\end{tabular}




\section{References}

[1] Groenwold AA, Stander N (1998) A 24-DOF fournode flat shell finite element for general unsymmetric orthotropic layered composites. Engineering Computations 15(4): 518-543.

[2] Bergan PG, Felippa CA (1985) A triangular membrane element with rotational degrees of freedom. Computer Methods in Applied Mechanics and Engineering 50: 25-69.

[3] Cook RD, (1986). On the Allman triangle and a related quadrilateral element. Computers \& Structures 22: 1065-1067.

[4] Long YQ, Xu Y (1994) Generalized conforming quadrilateral membrane element with vertex rigid rotational freedom. Computers \& Structures 52(4): 749-755.

[5] Pimpinelli G (2004) An assumed strain quadrilateral element with drilling degrees of freedom. Finite Elements in Analysis and Design 41: 267-283.
[6] Murat Y, Kömürcü S, Demirkan E. Geometrical nonlinear interaction model of thin insulating glass units. 13th International Congress on Advances in Civil Engineering, 12-14 Sept. 2018, Izmir, Turkey.

[7] Rydzewski JR. Theory of Arch Dams. Pergamon Press, Oxford, 1965.

[8] Bernardou M, Boisserie JM. The Finite Element Method in Thin Shell Theory: Application to Arch Dam Simulation. Springer, New York, 1982.

[9] Reddy JN. An Introduction to the Finite Element Method. McGraw-Hill, New York, 2005.

[10] Bhatti MA. Advanced Topics in Finite Element Analysis of Structures with Mathematica and MATLAB Computations. John Wiley \& Sons, Hoboken, New Jersey, 2006. 